\title{
Multi-stage Attack Detection and Signature Generation with ICS Honeypots
}

\author{
Emmanouil Vasilomanolakis, Shreyas Srinivasa, Carlos Garcia Cordero, Max Mühlhäuser \\ Center for Advanced Security Research Darmstadt (CASED) \\ Telecooperation Lab, Technische Universität Darmstadt \\ \{vasilomano,garcia,max\}@tk.tu-darmstadt.de, \{shreyas.srinivasa\}@ stud.tu-darmstadt.de
}

\begin{abstract}
New attack surfaces are emerging with the rise of Industrial Control System (ICS) devices exposed on the Internet. ICS devices must be protected in a holistic and efficient manner; especially when these are supporting critical infrastructure. Taking this issue into account, cyber-security research is recently being focused on providing early detection and warning mechanisms for ICSs. In this paper we present a novel honeypot capable of detecting multi-stage attacks targeting ICS networks. Upon detecting a multi-stage attack, our honeypot can generate signatures so that misuse Intrusion Detection Systems (IDSs) can subsequently thwart attacks of the same type. Our experimental results indicate that our honeypot and the signatures it generates provide good detection accuracy and that the Bro IDS can successfully use the signatures to prevent future attacks.
\end{abstract}

\section{INTRODUCTION}

The dependence of our society to IT networks is constantly increasing. In addition, the emergence and interconnectivity of Industrial Control Systems (ICSs) creates a plethora of security challenges that need to be addressed. Recent highly sophisticated and tailored attacks against these systems, e.g., Stuxnet [5] and Flame [16], are already here to highlight this fact.

Intrusion Detection Systems (IDSs) are nowadays considered a mandatory line of defense for the protection of critical networks [15]. The majority of IDSs in real world networks are passively monitoring and performing signaturebased detection. As such, the accuracy of these systems is highly influenced by the quality of the utilized signatures. Security mechanisms that exhibit more active monitoring can act as an additional line of defense by complementing existing systems. In this paper, we introduce a honeypot capable of providing such an active monitoring approach that can generate signatures of detected attacks on-the-fly.

Honeypots are systems whose only value is to be probed, attacked and compromised [11]. Their purpose is to attract malicious users, study their activities and, at the same time, reduce the attack surface. It is important to note that since honeypots do not feature any other purpose, by definition, any interaction with them is considered an attack. Thus, they do not exhibit false positives, i.e., all incoming traffic is considered malicious.

In this paper, we extend our previous work in the area of honeypots [13] with the focus of detecting sophisticated

978-1-5090-0223-8/16/\$31.00 (C) 2016 IEEE attacks in ICS networks. We specialize in the identification of attacks that originate from the same entity and make use of multiple protocols. We argue that these attacks can realistically describe cases of Advanced Persistent Threats (APTs) and targeted real world attacks. Our honeypot is able to extract signatures from detected attacks which can subsequently be imported into the Bro IDS [7]. We compare our solution with another honeypot from the related work, discuss the possibility of evading the honeypots and evaluate the quality and applicability of the generated signatures.

The remainder of this paper is as follows. In Section II, we present the related work in the areas of honeypots and alert generation. Section III provides an overview of the system as a whole; we give a formal model for the detection mechanisms and describe the signature generation process. Section IV presents and discusses the results of our evaluation. Lastly, Section V concludes this paper.

\section{RELATED WORK}

In this section we discuss related work in the areas of honeypots and signature generation with an emphasis on ICS networks.

In our previous work [13] we presented HosTaGe, an opensource low-interaction mobile honeypot. The idea was to introduce lightweight portable honeypots for mobile devices that aim at detecting malicious devices in wireless networks. HosTaGe supports the identification of attacks in all major protocols, e.g., HTTP, SMB, Telnet, FTP, MySQL, SIP and $\mathrm{SSH}$. We enhance our system in a twofold manner: first, we add support for ICS protocols and, second, we focus on detecting attacks triggered from the same entity and manifested in multiple protocols. In addition, we formalize such attacks via state machines and generate corresponding signatures for them to be used by IDSs.

In their recent work, Minn et al. [6] proposed IoTPOT, a honeypot that emphasizes on IoT devices by emulating the Telnet protocol. Their results show an increase of attacks on Telnet that target Internet of Things (IoT) devices, which also corresponds to our previous work's findings [14]. However, their focus is limited only into Telnet-based attacks (and different CPU architectures). Conpot [9] is another low interaction honeypot that focuses on emulating server side ICSs. Conpot was one of the first honeypots detecting ICS 
network attacks and is considered the state-of-the-art in this area. Conpot has a few disadvantages however; first, it does not support the emulation of Telnet, and the information that is logged, e.g., for Modbus attacks, is not always sufficient for an in-depth analysis of an attack. Moreover, as we will demonstrate in Section IV, Conpot can be identified as a honeypot by specialized tools. Nevertheless, as this honeypot is, with respect to its ICS support, the closest to ours, we compare these two systems in Section IV.

In the recent years there have been proposals in the area of signature generation via the utilization of honeypots [4], [12], [3]. For instance, HoneyComb [4] is a system that makes use of the honeyd honeypot [8] to generate alert signatures. It has the advantage of only using honeypot network traffic and thus reducing false positives. However, as a result of utilizing honeyd, only high level TCP or UDP information can be examined, making it unsuitable for payload-level analysis. Additionally, neither HoneyComb, nor any honeypot, is able to identify multi-stage attacks.

Finally, with regards to generic signature generation, in [10], Sengar et al. proposed a novel intrusion detection mechanism for Voice over IP (VoIP) that utilizes protocol-level state machines. Our approach is inspired by this work and builds on top of it. We move beyond VoIP and focus on attacks that target ICS networks. Our finite state machines are higher level as we focus on the detection of multi-stage (and thus multiprotocol) malicious activity. Furthermore, we utilize this state machine-based detection mechanism in our honeypot only as the initial step for improving intrusion detection. Our aim is to utilize the results gathered from Extended Finite State Machines (EFSMs) to automatically generate signatures and security policies that can be deployed in existing state-of-theart IDSs, i.e., Bro, rather than proposing a new IDS.

\section{HosTaGe ICS HONEYPOT}

In this section, we first give an overview of our system, discuss the ICS-specific protocols simulated by HosTaGe followed by the formal model used to describe the detection mechanisms we employ. Lastly, we discuss the signature generation of our proposal.

HosTaGe is a lightweight low-interaction honeypot for mobile devices. It is an open source project, written in Java, consisting of more than 15, 000 lines of code. As a low-interaction honeypot, HosTaGe simulates protocols in the TCP/IP stack, emulating many typical protocols utilized by adversaries, e.g., HTTPS, FTP, MySQL, SIP, SSH, etc. In order to support the protection of ICS networks, we modified, included and rewrote protocol detection mechanisms to follow the model we describe in this section. Moreover, we argue that a honeypot can be a supplemental security mechanism. Hence, we support such a task by generating signatures that can be automatically used in IDSs. A practical usage scenario can be the following. HosTaGe can be placed in the perimeter of a corporate network that, internally, includes ICSs. In the case of an adversary who aims at attacking the internal network, it is highly possible that the honeypot will be triggered during either the reconnaissance phase or the actual attack. If this is the case, $H o s T a G e$ will not only notify the network administrators but will also produce a signature for the attack and forward it to the internal IDSs.

\section{A. ICS Specific Protocol Emulation}

We extend the protocol emulation capabilities of HosTaGe by adding (or improving) support for protocols that are utilized in ICSs, i.e., Modbus, S7, SNMP, HTTP, Telnet, SMB and $S M T P$. In the following we briefly discuss these protocols and how they are emulated in our honeypot.

Among the various profiles supported by ICS devices, Modbus acts as a backbone for device communication. Modbus is a serial communications protocol initially published by Modicon for usage in its Programmable Logic Controllers (PLCs). It is now considered as the standard that connects industrial devices, and in particular it establishes communication between master and slave devices. As Modbus can also be used in a wireless mode for Remote Terminal Unit (RTU)-based communication, it is widely used in various ICS networks, e.g., nuclear power plants, gas and oil distribution, for communication between the PLCs.

a) Modbus: Modbus has instruction sets for the interaction of devices. PLCs have registers ${ }^{1}$ as memory units. The instruction sets are specified as functions which denote Read/Write (R/W) operations on the registers of the PLCs. Modeling the correct behavior of Modbus requires simulating the responses of the Modbus system for a command issued by a Modbus master device. HosTaGe simulates the Modbus communication protocols by implementing the request/reply mechanism. The Siemens PLCs also have registers as memory units, which store sensor data and application logic. The registers have the memory mapped to blocks at the register level. Through the Modbus protocol, the data in registers can be accessed and set. HosTaGe supports this request/reply paradigm for the memory mapped registers through dedicated data structures. Furthermore, the honeypot also supports the instruction set of Modbus, which is implemented as function codes. The instruction set involves registers' $\mathrm{R} / \mathrm{W}$, as well as the Modbus service diagnostics. Furthermore, in our Modbus implementation we aim on avoiding detection from wellknown reconnaissance tools. In more details, the Nmap port scanning tool, with special scripts, is able to perform Modbusspecific detection and reconnaissance. Similarly, the Metasploit exploit toolkit also provides support for the detection and exploitation of Modbus. HosTaGe is able to respond correctly in all these cases. In Section IV-C, we discuss the importance of this in the context of honeypot evasion attacks.

b) S7: The $S 7$ protocol (S7 Communication) is a Siemens proprietary protocol utilized in PLCs of the Siemens S7-300/400 families. It is used for PLC programming, exchanging data between PLCs, accessing PLC data from SCADA systems and for diagnostic purposes. The protocol forms as a base for accessing the registers for $\mathrm{R} / \mathrm{W}$ operations

\footnotetext{
${ }^{1}$ For simplicity we include coils in the term registers, even though strictly speaking they exhibit differences.
} 
and also programming the PLC for user defined tasks. The S7 protocol has been implemented to simulate the communication with the PLC with the memory mapping of the Siemens S7 300.

c) SNMP: The Siemens S7 family of PLCs supports the configuration of client devices through SNMP. This allows to remotely manage devices on the network. The SNMP protocol has been implemented (using the open source snmp4j library) to simulate an SNMP agent working on the Modbus slave profile and SNMP manager on the master profile.

d) HTTP: HTTP is supported by the majority of the PLCs for remote configuration purposes. The HTTP web server in the PLC enables GET/POST messages for information exchange. This HTTP server is simulated by HosTaGe through its dynamic HTTP protocol implementation. A default welcome page that simulates the configuration website of a PLC is displayed when an adversary tries to access port 80.

e) Telnet: The Telnet protocol allows accessing a basic shell on devices in order to read memory, delete files and execute commands. The Siemens S7 PLC also supports Telnet; users or applications can communicate with the PLC for file and backup operations. As such, HosTaGe supports this protocol by providing shell emulation for the attackers.

f) $S M B$ : The $S M B$ protocol enables network file sharing between devices of the same network. Previous analysis of the Stuxnet malware made evident that many attacks targeting ICSs make use of SMB to propagate. In Modbus, master systems control slaves and disseminate commands. These systems are usually control servers or host systems connected to PLCs or slaves that receive critical information and updates from the sensors placed on devices and PLCs. The master system is frequently a Windows XP host connected in a LAN. By emulating the SMB protocol, along with Modbus, it is possible to detect not only external attacks but also malicious activities originating from the Intranet, e.g., the propagation of Stuxnet. Thus, in HosTaGe the SMB protocol has been implemented and customized to simulate a Modbus master system. The customization involves using the SMB protocol to simulate $\mathrm{HosTaGe}$ as a shared network drive to which a malware might attempt to propagate after infiltrating a host machine. The propagated file is detected, by the so-called file injection module, and its hash value is sent to VirusTotal for further analysis.

g) SMTP: Lastly, SMTP is used as a notification system for ICSs; it is utilized to notify devices about changes that trigger tasks. The SMTP service implemented in HosTaGe does not provide the notification service, rather, it provides a very basic protocol emulation for simple service discovery.

In the remainder of this section we go deeper into the detection and signature generation mechanisms of HosTaGe. We first provide the fundamental formal model, in the form of EFSMs, followed by a discussion of the signature generation.

\section{B. Formal Model}

Our detection mechanism is formalized with an Extended Finite State Machine (EFSM). An EFSM has all the properties of a normal Finite State Machine (FSM) with the added feature of utilizing if-conditions, instead of only boolean conditions, to specify how a state transitions to a new state [2]. The formal model of our proposed detection mechanism is given by the Attack Detection EFSM $M=\left(S, s_{0}, I, O, V, P, \delta, \lambda\right)$ illustrated in Figure 1.

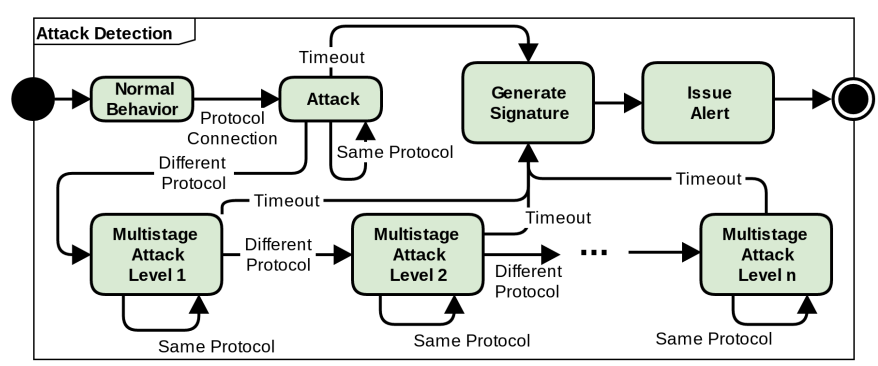

Fig. 1. EFSM of the attack detection and signature generation mechanism.

The set of all states are represented with $S$. The EFSM starts in the Normal Behavior state, represented by $s_{0}$. If any protocol communication is detected by the honeypot, the EFSM transitions to the Attack state. For as long as the same protocol attack is observed, the state remains the same. If a timeout occurs the EFSM transitions to the Generate Signature state followed by the Issue Alert state. The signature generation is optional and will capture either single attack or multistage attack types. After an initial attack, observing attacks originating from other protocols (but the same host) that have not yet been observed moves the state to the next Multistage Attack Level $x$, where $x$ corresponds to the number of different protocols observed after the first one.

The inputs $I$, outputs $O$, variables $V$ and predicates $P$ are tightly linked together. State transitions are carried out whenever specific inputs $i \in I$ are received. This transitions may also generate an output $o \in O$. In the Normal Behavior, Attack and Multistage Attack Level $x$ states, the supported protocols are used as inputs and outputs. As such, \{Modbus, S7, SNMP, HTTP, Telnet, SMB, SMTP, HTTPS, SSH, FTP $\in I \in O$ for these states. The inputs $I$ are not limited, however, to only ports. Special activities of interest on a protocol are also considered inputs. For instance, the act of requesting a file through the $S M B$ protocol (see Section III-A) is an input on itself. $V$ is a finite set of variables. These variables are used to construct a set of predicates $P$ used for determining if a state transitions to another one. Each attack state hold a boolean variable $v \in V$ for each emulated port. If a particular port has been observed in the entire life of the EFSM, the corresponding variable for that port will be set to true. Besides variables, predicates $P$ consist of the logic operator $A N D$ and the arithmetical operator $=$. We define the Protocol Connection predicate as the condition where a new protocol is observed without having observed other protocols yet. The Different Protocol predicate indicates, as the name suggests, that a new protocol has been observed after having seen at least one other. If any of these predicates is true a state transition takes place. 
The final element of our model is the set of transitions $\delta\left(s_{i}, i, p\right)=s_{j}$ and the outputs $\lambda\left(s_{i}, i, p\right)=o$ generated by the transition itself. The set of transitions specify that whenever state $s_{i} \in S$ receives the input $i$ and the predicate $p \in P$ is satisfied, the EFSM transitions to state $s_{j}$ and outputs $o \in O$. The outputs are used by the Generate Signature state to create signatures for misuse analysis.

\section{Detection Mechanisms in HosTaGe}

HosTaGe can distinguish between three different classes of attacks: Single-Protocol Level Detection (SPLD), Multi-Stage Level Detection (MSLD) and Payload Level Detection (PLD).

SPLD attacks refer to those that occur on a single protocol, e.g., HTTP connection attempts without observing other protocols or any extraordinary payload-level information. This is the simplest type of detection which still contains interesting analysis potential. For example, we can infer that a multitude of malware is trying to exploit the Telnet protocol (see Section IV) due to the increasing amount of IoT devices such as IP Webcams. As this is the simplest of the three cases, we omit the description of its respective EFSM due to space constrains. In fact, the EFSM shown in Figure 1 can be seen as a generalized example of MSLD.

MSLD refers to attacks that originate from the same source and attempt to exploit different types of protocols within a small window of time. These type of attacks are identified by our honeypot with the EFSM shown in Figure 1, as described in Section III-B. An important factor in MSLD is the timewindow $(t w)$ that determines whether an attack should be mapped as the SPLD or the MSLD class. This means that when the EFSM is on the Attack state and no further activity is detected (for a maximum of $t w$ ) a timeout will occur and the attack will be identified as SPLD. The $t w$ can be adjusted with respect to the monitored network and its requirements. In this paper (see Section IV), we experimented with the $t w$ value of 15 minutes. In practice, this suggests that when $t w=15$, a transition from the Attack to the Multistage Attack Level 1 state (via the Different Protocol predicate) is possible when a new attack occurs within the specified $t w$.

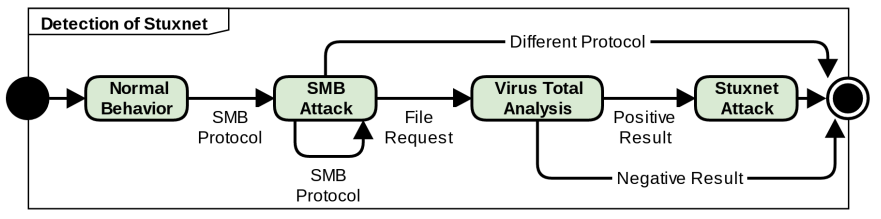

Fig. 2. EFSM for PLD in the case of Stuxnet propagation

PLD extends the applicability of the EFSM with respect to the input $I$. Referring back to our formal model, the outputs $o \in O$ from the Attack and Multistage Attack Level $x$ states are used in the Generate Signature state to create signatures. Signatures are also EFSMs that comply with the presented model. As we already mentioned, the input is not limited only to a port or protocol but also to potentially interesting payload-level information. Take Figure 2 as an example of an
EFSM that represents a signatures generated by PLD. This signature identifies Stuxnet attacks from the set of outputs $O$ obtained from the Attack Detection EFSM shown in Figure 1. The Detection of Stuxnet EFSM assumes an initial Normal Behavior state and transitions to SMB Attack if an SMB protocol is observed. Stuxnet tries to inject an infected file through SMB. After a file is received, it (or its hash value) is sent to VirusTotal and, if the file is indeed malicious, the EFSM transitions to the Stuxnet Attack state in which the presence of Stuxnet is reported.

\section{Signature Generation}

As discussed in the previous section, upon detecting an intrusion, HosTaGe is able to generate signatures for the Bro IDS. IDSs usually inspect all incoming packets for malicious content and make use of signatures to determine whether traffic is malicious or not. HosTaGe captures not only the attack packets, but also all received connection requests at the packet level. In addition, it records the entire connection tear-down that takes place between an adversary and the honeypot. HosTaGe utilizes specific modules to handle the above features, i.e., the listener, logger, connection guard, HosTaGe service, multistage attack detection, and file injection detection modules. The Bro IDS uses the so-called Bro language, an event-driven scripting language that can be used for extending and customizing the IDS's functionality. Bro's signatures rely on packet data to check for the content to be matched for incoming packets. The signature generation module in $\mathrm{HosTaGe}$ is used to generate the signature files ${ }^{2}$ for Bro. By specifying the protocols for the signature generation mechanism, the packet information of the connection are derived through the attack records module from HosTaGe to a template signature file which is used to generate signature files for Bro. The attack record module of HosTaGe generates signature files for a protocol.

An example of a signature generated by HosTaGe, for the Modbus protocol, is shown in the Listing 1. This signature, automatically created from HosTaGe and written in the Bro language, is able to detect the well-known Metasploit script for Modbus services identification.

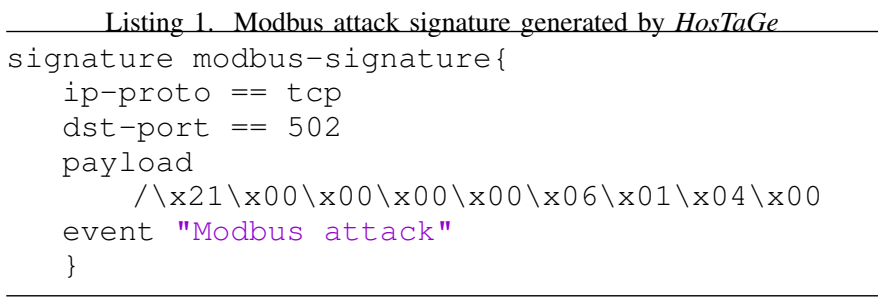

\section{RESULTS}

In this section we show various experiments that demonstrate HosTaGe's detection capabilities as well as its ability to generate signatures from detected attacks. In addition, we

\footnotetext{
${ }^{2}$ Utilizing the Bro terminology, HosTaGe can generate security policy files.
} 
discuss observed multi-stage attacks and give some insights regarding the detectability of our honeypot.

\section{A. Honeypot Comparison}

In this section, the HosTaGe and Conpot honeypots will be compared. Both honeypots were deployed in a controlled environment with no firewalls in between the honeypots and the Internet. The honeypots had similar IP addresses in the sense that they were in the same $/ 24$ subnet. Note that the IP addresses were dynamic, hence, offering more generic observations for the two honeypots. The evaluation period for our tests was 12 weeks for all protocols except for $S 7$ that was limited to eight weeks. We should note that the purpose of this experiment is to compare the two honeypots and to identify automated attacks that target ICS networks; thus, no advertisement of the honeypots was made. However, as we later show, both honeypots were probed by a certain search engine (Shodan).
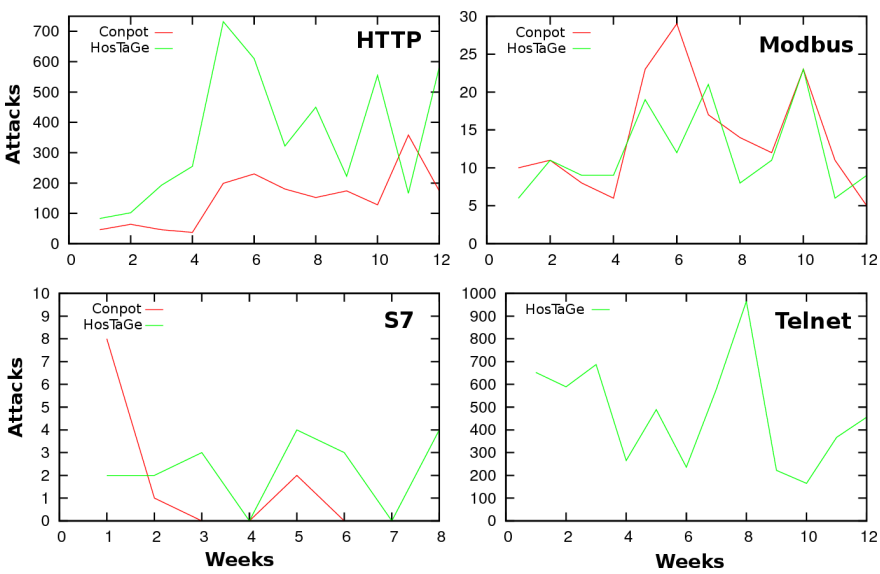

Fig. 3. Comparison of attacks on HosTaGe and Conpot for HTTP, Modbus, $S 7$ and Telnet. Note, that Conpot does not support the Telnet protocol.

The results of our analysis are given in Figure 3. We compare the results gathered from the two honeypots for the HTTP, Modbus and $S 7$ protocols. In addition, we present the results gathered for Telnet (even though Conpot does not support it) as Telnet is considered an important attack factor for ICSs networks [6]. Finally, note that the $S 7$ protocol comparison presented in the figure is for a shorter period of time (i.e., 8 weeks) as $S 7$ 's implementation in $H o s T a G e$ was completed in a later stage. As one can observe from the results, HosTaGe exhibits good detection accuracy in comparison to Conpot. In fact, in most cases HosTaGe detected more attacks than Conpot (e.g., HTTP, S7), while for the case of Modbus the number of detected attacks is almost equivalent.

Lastly, Figure 4 depicts the malicious unique IP addresses (and also their intersection) that were detected for each honeypot (for the HTTP and Modbus protocols). In a glance, the histogram shows that $\mathrm{HosTaGe}$ was able to consistently detect more unique IP addresses (of attackers) than Conpot.

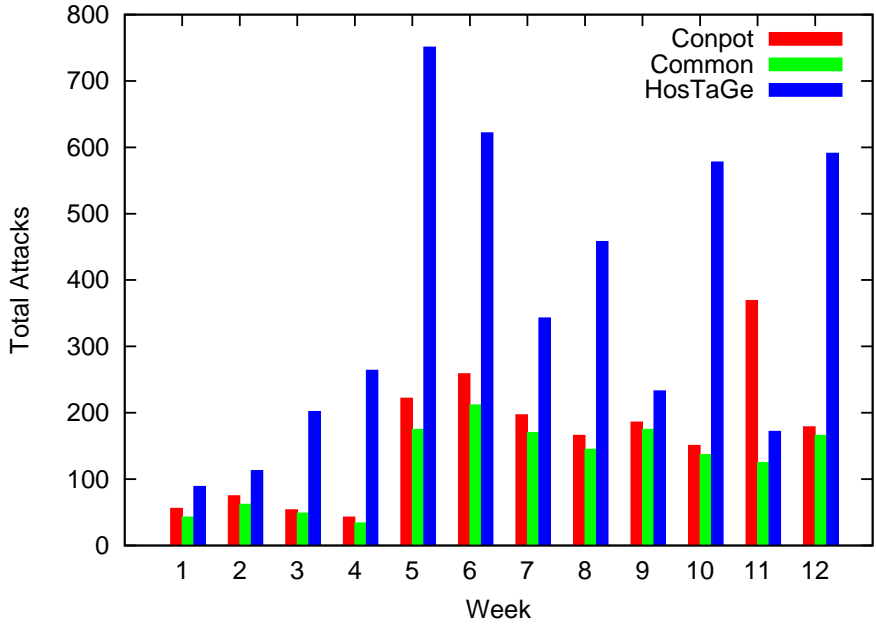

Fig. 4. Comparison of unique and common malicious IP addresses targeting HosTaGe and Conpot

\section{B. Multi-Stage attacks}

During the observed period, HosTaGe also automatically detected three multi-stage ICS attacks (within a time-window of $t w=15$ minutes). First, an attack from Iran was detected, which based on the Geo-IP information, appeared to be close to where Stuxnet was initially detected, i.e., the Tehran Nuclear Research Center. The attack included a portscan and sent an HTTP GET request to the honeypot. Subsequently, we detected an Nmap script that was trying to validate whether a Modbus service is indeed running on the host. The second interesting multi-stage attack was originated from the Zhejiang Nuclear Industry in China. It started with a portscan, continued with an HTTP attack and finished with Modbus protocol requests that queried specific ICS services. The last attack was from an area close to a power plant in Colombia. The attack stages consisted of a web-connection through HTTP, a general purpose scan of the network and finally an attempted Telnet connection to the honeypot.

\section{Honeypot Evasion}

One essential requirement for honeypots is their ability to remain undetected; hiding the fact that this is indeed a honeypot and not what is being advertised. In this section we discuss how, in the aforementioned experimental setup, HosTaGe managed to remain undetected (while Conpot did not) from the Shodan ${ }^{3}$ search engine. Shodan is a specialized search engine that crawls the Internet and attempts to identify connected devices, e.g., IP web-cameras, ICSs, etc. [1]. Through the continuous process of crawling and indexing, the system creates an up-to-date database of systems and services exposed on the Internet. Recently, Shodan implemented detection mechanisms to identify honeypots. It performs a series of probes, and checks, and subsequently creates a score for each probed device. Based on this score value, Shodan determines whether a specific system is an honeypot or not.

\footnotetext{
${ }^{3}$ https://www.shodan.io
} 
A few weeks after our initial experiments, both $\mathrm{HosTaGe}$ and Conpot received probes from IPs that belong to Shodan. The protocols probed were S7, Modbus, SSH and HTTP, among others. For the SSH and HTTP protocols the messages and requests were of low complexity; the HTTP protocol involved a GET request and the SSH an attempt to establish a connection. The S7 and Modbus attacks were more complicated. Our analysis indicates that the probes are most likely the result of modified Nmap/Metasploit scripts for ICSs identification. These probes were able to identify Conpot as a honeypot but not HosTaGe. The S7 attack involved an audit of the device type, location, serial number, plant identification and module name. The Modbus attack involved fetching details of certain units (i.e., unit number 0 and 255) and their slave data. On the one hand, Conpot could not respond meaningfully in all the aforementioned requests (either due to static serial numbers or Modbus protocol simulation errors) and thus it was classified as a honeypot. On the other hand, HosTaGe managed to respond successfully and hence remain undetected.

\section{Signature Generation}

The last part of our evaluation focuses on the signature generation mechanism and its effectiveness. We study the applicability of the generated signatures.

Our first goal is to generate multi-stage attack signatures. To achieve this we manually inject attacks into a HosTaGe instance in a controlled environment. Attacks are additionally captured by the network packet capture tool Wireshark and saved for future reference (see below). Simultaneously, HosTaGe detects attacks and constructs signatures from both the protocol and payload-level interaction.

For determining the applicability of the generated signatures, we perform two different tests. All tests utilize publicly available datasets of network traffic (in the form of pcap files). They consist of synthetic and real network captures ${ }^{4}$, malware focused traffic ${ }^{5}$, and honeypot captured traffic ${ }^{6}$

First, we determine whether the generated signatures detect false positives. We import the signatures into the Bro IDS and replay the network traffic of the (unmodified) test datasets. With respect to our definitions in Section III-C, we utilize a time-window of $t w=15$ (minutes) for our tests. No multistage attacks were detected by Bro, as expected. Afterwards, we merge each dataset with the network traffic captured by Wireshark in the initial step (that includes our injected multistage attacks). Subsequently, we replay each modified network file while Bro is running. In all cases, Bro successfully detects all the injected attacks without generating any false positives.

\section{CONCLUSION}

The increasing number of cyber-attacks, along with the need to protect critical infrastructure, such as ICSs, highlights the need for tailored security mechanisms. In this paper we

\footnotetext{
${ }^{4}$ Small and Big flows datasets: http://tcpreplay.appneta.com/wiki/captures.html

${ }^{5}$ CTU-13 Dataset: https://stratosphereips.org/category/dataset.html

${ }^{6}$ HoneyBot Dataset: http://www.netresec.com/?page=PcapFiles
}

presented a honeypot capable of detecting attacks on ICS networks. We formalize the detection mechanisms with EFSMs and discuss the ability to automatically generate signatures from observed attacks. Our experiments show that our proposal can be easily deployed in practice and that it is able to support existing intrusion detection infrastructure. As future work, we plan to further examine both honeypot evasion mechanisms as well as honeypot-side techniques to avoid such attacks. Lastly, we aim at further assessing Shodan's impact on the effectiveness of a honeypot (after its identification), as malware might make use of this information to avoid contacting well known honeypots.

\section{REFERENCES}

[1] Roland Bodenheim, Jonathan Butts, Stephen Dunlap, and Barry Mullins. Evaluation of the ability of the Shodan search engine to identify Internetfacing industrial control devices. International Journal of Critical Infrastructure Protection, 7(2):114-123, 2014

[2] Marcelo Fantinato and Mario Jino. Applying extended finite state machines in software testing of interactive systems. In Interactive Systems: Design, Specification, and Verification (DSV-IS), volume 2844, pages 34-45. Springer Berlin Heidelberg, 2003.

[3] Hyang-Ah Kim and Brad Karp. Autograph: Toward automated, distributed worm signature detection. In USENIX security symposium, volume 286. San Diego, CA, 2004.

[4] Christian Kreibich and Jon Crowcroft. Honeycomb - Creating Intrusion Detection Signatures Using Honeypots. In ACM SIGCOMM Computer Communication Review, volume 34, pages 51 - 56, 2004.

[5] R Langner. Stuxnet: Dissecting a cyberwarfare weapon. Security \& Privacy, IEEE, (June):49-51, 2011.

[6] Yin Pa Minn, Shogo Suzuki, Katsunari Yoshioka, Tsutomu Matsumoto, and Christian Rossow. IoTPOT : Analysing the Rise of IoT Compromises. In 9th USENIX Workshop on Offensive Technologies (WOOT). USENIX Association, 2015.

[7] Vern Paxson. Bro: a system for detecting network intruders in real-time. Computer Networks, 31(23-24):2435-2463, dec 1999.

[8] Niels Provos. Honeyd : A Virtual Honeypot Daemon. In DFN-CERT workshop, 2003.

[9] Lukas Rist, Daniel Haslinger, John Smith, Johny Vestergaard, and Andrea Pasquale. Conpot honeypot, 2013.

[10] Hemant Sengar, Duminda Wijesekera, Haining Wang, and Sushil Jajodia. VoIP intrusion detection through interacting protocol state machines. In International Conference on Dependable Systems and Networks (DSN), pages 393-402. IEEE, 2006.

[11] Lance Spitzner. Honeypots : Catching the Insider Threat. In Computer Security Applications Conference, pages 170-179. IEEE, 2003.

[12] Urjita Thakar, Sudarshan Varma, and AK Ramani. Honeyanalyzeranalysis and extraction of intrusion detection patterns \& signatures using honeypot. In Proceedings of the Second International Conference on Innovations in Information Technology, 2005.

[13] Emmanouil Vasilomanolakis, Shankar Karuppayah, Mathias Fischer, Max Mühlhäuser, Mihai Plasoianu, Lars Pandikow, and Wulf Pfeiffer. This Network is Infected : HosTaGe - a Low-Interaction Honeypot for Mobile Devices. In Security and privacy in smartphones \& mobile devices, pages 43-48. ACM, 2013.

[14] Emmanouil Vasilomanolakis, Shankar Karuppayah, Panayotis Kikiras, and Max Mühlhäuser. A honeypot-driven cyber incident monitor: lessons learned and steps ahead. In International Conference on Security of Information and Networks. ACM, 2015.

[15] Emmanouil Vasilomanolakis, Shankar Karuppayah, Max Mühlhäuser, and Mathias Fischer. Taxonomy and Survey of Collaborative Intrusion Detection. ACM Computing Surveys, 47(4):33, 2015.

[16] Nikos Virvilis and Dimitris Gritzalis. The Big Four - What We Did Wrong in Advanced Persistent Threat Detection? In International Conference on Availability, Reliability and Security, pages 248-254. IEEE, 2013. 